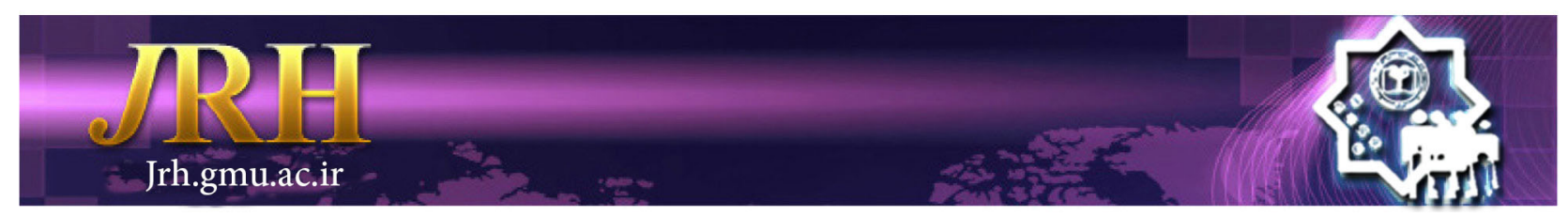

\title{
Reliability, validity and factor structure for the attitudes toward infidelity scale
}

Mojtaba Habibi ${ }^{1}$, Sayed Hadi Sayed Alitabar ${ }^{2}$, Minoo Pouravari ${ }^{3}$, Somaieh Salehi ${ }^{4}$

\author{
Journal of Research \& Health \\ Social Development \& Health Promotion \\ Research Center \\ Vol. 9, No.4, Jul \& Agu 2019 \\ Pages: 294- 301 \\ DOI: 10.29252/jrh.9.4.294 \\ Original Article
}

1. Department of Family Therapy, Family Research Institute, Shahid Beheshti University, Tehran, Iran

2. Correspondence to: Department of Career Counseling, Faculty of Psychology \& Educational Sciences, University of Isfahan, Isfahan, Iran

Email: hadialitabar@gmail.com

3. Department of Counseling, Faculty of Psychology \& Educational Sciences, Alzahra University, Tehran, Iran

4. Department of Psychology, Faculty of Psychology \& Educational Sciences, Shahid Beheshti University, Tehran, Iran

Received: 21 Feb 2018

Accepted: 21 May 2018

How to cite this article: Habibi M, Sayed Alitabar SH, Pouravari M, Salehi S. Reliability, validity and factor structure for the scale of attitudes toward infidelity. $J$ Research \& Health2019; 9(4): 294- 301.

\begin{abstract}
Infidelity is a harmful and a main problem in the therapy of couple relationships. The present study examined the reliability, validity and factor structure of the attitudes toward the infidelity scale among the patients referred to counseling centers in Tehran. 528 participants (185 females and 343 males) were selected by convenience sampling method from 5 centers. The study instrument was Whately's attitudes toward infidelity scale, which translated by 7 psychologists \& back translated by an English expert. After a pilot study on 30 participants, the necessary corrections were made. The structure of the scale factor was confirmed in a first-order factor using confirmatory factor analysis. This scale had a good internal consistency (Cronbach's alpha $=0.71$ and test-retest $=0.87$ ) It had correlation with Alport internal and external religious orientation scale respectively 0.29 and 0.16 , also negative correlation with Kansas Marital satisfaction scale and Enrich sexual satisfaction subscales ( -0.22 and -0.16 respectively). This study showed that ATIS is an efficient tool for research and couple counseling and has an appropriate validity and reliability for Iranian society.
\end{abstract}

Keywords: Attitude, Marriage, attitudes toward infidelity scale

\section{Introduction}

At present, infidelity is considered harmful and as a main problem in the therapy of couple relationships [1]. It is also the cause of most divorces, and some evidences indicate that such relationships are increasing similarly between men and women [2-5]. In this regard, the attitude toward infidelity has emerged as a significant variable in the majority of researches. People with easier attitude to extramarital relations, are more likely involved in such relationship [4,6,7]. Also Buunk and Bakker [8] found that attitude toward extramarital sex is an important index to predict the willingness toward it. The beliefs and values encourage or prohibit the engagement in extramarital relations. In this regard, gender not only runs as the originator process, but also significantly conveys different beliefs and values to men and women [9-11]. According to interpersonal variables, men have more positive attitude toward extramarital relations compared with women and experience it more $[3,5,10,12,13]$. In addition, men with easier attitude to infidelity are more likely involved in this kind 
of relationship [7,14]. As a result, men are more likely to believe that infidelity relations are normal or at least acceptable. Also for women, easy-going sexual attitude is the best way to predict infidelity among them [15]. Age and gender are the mediator variables, although it seems data are contradictory. Treas and Giesen [4] in this regard found that when the attitude or desire toward having easy going sex was controlled, the main effects of gender on extramarital relations reduced significantly. In another example, Atkins showed that gender is an important mediator variable in the prevalence of marital infidelity.

Education also may interfere in infidelity; however, findings are contradictory in this area. Some studies show that infidelity is more likely among people with lower education [16], while others report marital infidelity more among educated people [12]. Forste and Tanfer also believe that higher education can indicate looser sexual attitudes. For example, women who have more education than their partners are 3.6 times more likely to have such affairs than others [17]. Whatley designed the scale of ATIS to check the attitudes toward extramarital relationships and used it on 286 (150 males and 136 females) responders. Attitudes and opinions related to extramarital relationships were assessed on a survey using the first questionnaire with 51 items [18]. These items included the questions like "disloyalty would not harm anyone," and "if I knew my partner does not aware of the infidelity relationship, I had done it". Respondents responded to each question on a 7-point scale (Likert) 1 (extremely disagree) to 7 (extremely agree). The items which were phrased negatively were scored reversely, because higher scores showed more positive attitudes toward infidelity. Factor analysis was done by using maximum likelihood and varimax rotation. Factor analysis revealed that there were factors with eigenvalues greater than 0.1. After reviewing the Scree curved, singlefactor solution was considered as appropriate. For more flexibility in the study, 12 items were selected: 6 items were classified as positive and other 6 as negative items. Retest reliability (internal consistency) of this scale was estimated 0.80 . The factor of this scale was called "marital infidelity" and calculated for the $19.24 \%$ of the variances. The mean value and standard deviation of this factor were 27.85 and 12.02 respectively. Coefficient of variation was equal to 0.43 [18]. This estimated value shows the amount of variation in the scale which leads to diagnose or distinct high scores from low among individuals [19]. The higher value means the better characteristic of the instrument.

To determine the structure validity of the scale, the point biserial correlation coefficient was used among the scores of the attitude to extramarital relationships and true/false response of the participants to the question of "I never betrayed my partner". The results of the analysis were significant. Those who obtained higher scores in attitudes towards extramarital relationships were more likely to betray their partners. One-way analysis of variance (ANOVA) was also significant in determining the difference between two genders in regard of marital infidelity. In general, men had more positive attitudes toward infidelity compared with women [18].

Few studies have been conducted on the issues around infidelity in Iran which may be due to the lack of efficient tests in this field. The importance of this study is because of the applying such scale to examine the individuals' attitudes toward infidelity and predict their behavior in the field indirectly. In the present study, the scale of attitude toward infidelity was translated in order to assess its validity and reliability. It helps the interested researchers examine such attitudes in different classes and detect their associated factors. The aim of this study was to evaluate the reliability and validity for the scale of attitude toward infidelity in Iranian society.

\section{Method}

The population included all married men and women who were referring to counseling centers of Tehran and were at least were married for one year. Out of 9 counseling 
center in the zone of Tehran, 5 counseling centers were randomly selected. The study sample included 528 participants (185 females and 343 males) using convenience sampling method. In this study, Cohen's d formula was used to obtain an appropriate sample size [19]. The survey method (questionnaire) was used to collect data and it was conducted by the researcher and his colleagues in person. Before delivering the questionnaire, a description was presented on the way to respond the questions, the goal and necessity of honest cooperation. In addition, the participants were assured that their responses would be confidential and if they had provided their email addresses they would get their results at the end of the study. The given time to respond the questions was approximately 10 to 15 minutes.

Data collection was implemented through four instruments as follows:

Attitudes toward infidelity scale: As mentioned above this scale was made by Whatley [18] with 12 items and was scored on a seven point scale (Likert) from 1 (extremely disagree) to 7 (extremely agree). The study of Whatley [18] demonstrated a good reliability and validity of the scale. The Cronbach's alpha coefficients of internal consistency were assessed 0.80 in his study.

Religious orientation scale: Allport and ross developed this scale in 1950 to measure the external and internal religious orientation. The questionnaire consisted of 20 items. The internal religion is pervasive organized and internalized, while the external religion is used to satisfy individual needs, such as security and position. The initial studies on this basis showed that the correlation of external with internal orientation was 0.21 [20]. In Iran, the internal consistency was 0.71 , by using the coefficient of Cronbach's alpha. Besides, the retest reliability was 0.74 [21].

Kansas marital satisfaction scale (KMSS): This scale was made by Schumm et al. [29] and is widely used in the studies [22-24]. This scale has only three items. Despite the shortness, this scale has a good divergent validity [25]. Schumm et al. [29] found that Kansas Marital
Satisfaction Scale has good concurrent validity with Dyadic Adjustment Scale (DAS) [26] and Quality Marriage Inventory (QMI) [27]. Specifically Schumm et al. [29] reported $\mathrm{r}=$ 0.83 and $p<0.001$ by conducting KMSS and DAS for women and $r=0.91$ by conducting KMSS and QMI. Weigel, Ballard- Reich [28] in their study reported the internal consistency coefficient (Cronbach's alpha) of 0.97 in men and women by using Kansas. Schumm et al. on the study of married couples [29] reported alpha between 0.84 and 0.98 . The cronbach's alpha of this test in Iran was 0.92 .

Enrich sexual satisfaction: The 10-item scale of enrich sexual satisfaction was used to measure the sexual satisfaction [30]. This 10item scale measures the feelings of sexual and emotional relationship. These items reflect the attitudes toward sex, sexuality and sexual fidelity. Olson, Fournier and Drukman [30], achieved $\mathrm{r}=0.85$ for the internal reliability coefficient (Cronbach's alpha) and $r=0.92$ for retest reliability (with an interval of four weeks) of the scale of sexual relations. The subscales of sexual relationship its quality and conflict resolution are considered as the three main predictors of marital satisfaction among the 10 subscales of the Enrich sexual satisfaction in assessing the divergent validity for the diagnosis of satisfied individuals from dissatisfied ones [31].

Attitudestowardinfidelityscaleweretranslated from English to Persian then the Persian translation was back translated into English by another person. Next, two English versions were compared to detect the differences between them. The final version of translation was reviewed by 7 experts in psychology and counseling. These individuals assessed the perceptible degree of the questions and appearance of the questionnaire and whether the questionnaire has covered all aspects of the issue. The questionnaire items were read one by one in a focus group consisted of 10 married men and 10 married women, and all ambiguities were reviewed and amended. Then, the questionnaire was distributed between married men and women that were 
married for at least a year. Confirmatory factor analysis, univariate analysis of variance, t-test and Pearson's correlation coefficient were used to analyze data by using the software of SPSS16 and LISREL 8.72.

\section{Results}

Demographic distribution showed that the minimum and maximum age of the participants were between 20 and 68 years ( mean $=60.31$ and $\mathrm{SD}=48$ ) and 185 of the participants were female $(1.35 \%)$. Besides, the education of 94 persons was secondary-school education and Diploma (9.17\%), 279 persons Diploma and Associate's degree $(8.52 \%)$, and 155 Masters and above (3.29\%) respectively.

Prior to the examination of model fitness, assumptions were investigated, including: 1) normality of variables distribution, 2) multiple observed variables (at least two observed variables for each latent variable), 3) model with over identification, 4) if the measurement scale is interval [32]. Also, in the present study, their examination and observation were approved. Observing the first assumption was evaluated with respect to the results of univariate and multivariate normality in LISREL software. Due to the rejection of the normality hypothesis in some variables, the robust estimation method was used against violations of normality which is discussed in the section of parameter estimation. The LISREL software outputs, and fitting measurement models showed that the pre-assumptions of "over identified model" and "lack of multi-co-linearity" have been considered among variables. Finally regarding the nature of data the assumption of interval scale was confirmed.

The statistical fitness of the measurement model of the study data was examined by using software LISREL-8.72 [33]. The model consisted of one-dimensional model [18], which loaded 12 questions on the factor of scale for attitude toward infidelity. To evaluate the significance of parameters and the explanation coefficient of parameters, the factor loadings, standard errors of parameter estimates and t-test are presented in Table 1. Due to the violations of the normality assumptions, the method of robust maximum likelihood was used versus the violation of the normal distribution assumption to estimate the model. The following criteria were used for model fitness: The scale of satorra-bentler chisquare $\left(\chi^{2}\right)$, the ratio of chi-square to degrees of freedom $\left(\mathrm{df} / \chi^{2}\right)$, Goodness of Fit Index (GFI), Adjusted goodness of fit index (AGFI), Comparative Fit Index (CFI), Root Mean Square Error of Approximation (RMSEA) and Root Mean Square Residual (RMR).

Table 1 The results of confirmatory factor analysis for the scale of the Attitudes toward infidelity

\begin{tabular}{|c|c|c|c|c|}
\hline Items & $\begin{array}{c}\text { Factor } \\
\text { loadings }\end{array}$ & S.E & $\mathrm{t}$ & $\mathrm{R}_{2}$ \\
\hline Being unfaithful never hurt anyone. & 0.53 & 0.08 & 6.35 & 0.28 \\
\hline Infidelity in a marital relationship is grounds for divorce. & 0.69 & 0.07 & 9.51 & 0.48 \\
\hline Infidelity is acceptable for retaliation of infidelity. & 0.45 & 0.09 & 4.93 & 0.20 \\
\hline It is natural for people to be unfaithful. & 0.71 & 0.07 & 9.55 & 0.50 \\
\hline Online/internet behavior (e.g., sex chat rooms, porn sites) is an act of infidelity. & 0.53 & 0.12 & 4.34 & 0.28 \\
\hline Infidelity is morally wrong in all circumstances regardless of the situation. & 0.94 & 0.09 & 10.47 & 0.88 \\
\hline Being unfaithful in a relationship is one of the most dishonorable things a person can do. & 0.85 & 0.08 & 11.34 & 0.72 \\
\hline Infidelity is unacceptable under any circumstances if the couple is married. & 0.87 & 0.10 & 8.79 & 0.76 \\
\hline I would not mind if my significant other had an affair as long as I did not know about it. & 0.69 & 0.09 & 7.33 & 0.48 \\
\hline It would be acceptable for me to have an affair, but not my significant other. & 0.82 & 0.08 & 10.17 & 0.67 \\
\hline I would have an affair if I knew my significant other would never find out. & 0.88 & 0.07 & 11.86 & 0.77 \\
\hline If I knew my significant other was guilty of infidelity, I would confront him/her. & 0.42 & 0.11 & 3.81 & 0.18 \\
\hline
\end{tabular}

Table1 presents the results of confirmatory factor analysis for the scale of attitudes toward infidelity in terms of loadings fact or (Probable. Error), standard error of 
estimated loading factor, $\mathrm{t}$ value to evaluate the significance of loadings factor and the coefficient of determination for each question on the relevant factor $\left(\mathrm{R}_{2}\right)$.

The difference of the model fitness suggests that model fit data well. It means that the results of the study tend to support one-factor model. The indices of model fitness showed that the model fit data reasonably well. If the chi-square test is not statistically significant, it indicates a very good fit. However, this index is mostly significant in the samples larger than 100 , so, it is not a good index to measure model fitness. If the ratio of chi-square to the degree of freedom is less than 3 , it indicates a good fit. If the CFI, AGFI and GFI indices are greater than 0.95 and
RMR and RMSEA indices are smaller than 0.50 , it implies a very good and desirable fit. If CFI, AGFI, GFI indices are greater than 0.90 and RMR and RMSE indices are smaller than 0.80 , it implies an optimal and good fit [34-36]. Thus, CFI, AGFI, GFI indices in the current study imply a desirable and appropriate fitness. RMR and RMSEA indices also imply a desirable and appropriate fitness. Besides, the model fitness is satisfactory, based on the index of chi-square on the degree of freedom (Table 2). Therefore, based on the results of the first time measurement model we conclude that the measurement models of attitudes toward infidelity scale, has a suitable validity to be used in the studied community.

Table 2 The indexes of conformity factor analysis for the scale of attitudes toward infidelity

\begin{tabular}{cccccccc}
\hline RMR & RMSEA & CFI & AGFI & GFI & df $/ \chi 2$ & Df & $\begin{array}{c}\chi^{2} \text { Satorra- } \\
\text { bentler }\end{array}$ \\
\hline 0.12 & 0.047 & 0.97 & 0.93 & 0.95 & 1.74 & 52 & 90.67 \\
\hline
\end{tabular}

For evaluating the validity of this test, the structure validity (divergent validity) has been used. For this purpose, Religious orientation scale of Allport,
Kansas marital satisfaction, and Enrich sexual satisfaction subscale were applied. The values of divergent validity are presented in Table 3.

Table 3 The divergent validity for the scale of attitudes toward infidelity

\begin{tabular}{lcccc}
\hline Factor & $\begin{array}{c}\text { Internal religion } \\
\text { orientation }\end{array}$ & $\begin{array}{c}\text { External religion } \\
\text { orientation }\end{array}$ & $\begin{array}{c}\text { Kansas marital } \\
\text { satisfaction }\end{array}$ & $\begin{array}{c}\text { Enrich sexual } \\
\text { satisfaction }\end{array}$ \\
\hline $\begin{array}{l}\text { Attitudes toward } \\
\text { infidelity }\end{array}$ & $-0.29^{* *}$ & $-0.16^{* *}$ & $-0.22^{* *}$ & $-0.16^{*}$ \\
\hline$*_{p}<0.05, * * \mathrm{p}<0.01$ & & & &
\end{tabular}

Table 3 shows that the attitudes toward infidelity scale were significantly correlated with allport religious orientation, kansas marital satisfaction and enrich satisfaction sub-scale.

To determine the reliability of the scale two methods of cronbach's alpha and test-retest coefficient were used. cronbach's alpha coefficient for the scale of attitudes toward Infidelity was estimated 0.71 . As well, the test retest coefficient of conducting this test was measured 0.87 . To investigate this interaction, the relation between age and the attitudes toward infidelity Scale were examined using pearson's correlation coefficient.

Table 4 The mean difference in the attitudes toward infidelity among married men and women

\begin{tabular}{ccccccccc}
\hline Variable & $\begin{array}{c}\text { Statistic } \\
\text { group }\end{array}$ & $\begin{array}{c}\text { Sample } \\
\text { size }\end{array}$ & Mean & SD & t & df & p \\
\hline $\begin{array}{c}\text { Attitudes toward } \\
\text { infidelity }\end{array}$ & Men & 343 & 45.08 & 7.52 & -2.84 & 301.79 & 0.005 \\
\cline { 2 - 8 } & Women & 185 & 42.71 & 9.85 & & & \\
\hline
\end{tabular}

Based on the results, the value of $r$ with respect to the relation between age and the Attitude towards Infidelity Scale was not significant $(\mathrm{N}=528, \mathrm{r}=0.06)$. So it can be said that there 
was no significant relationship between these two variables. The results in Table 4 shows that there was no significant difference in terms of attitudes towards Infidelity among married male $(M=45.08)$ and women $(M=42.71)$ $(\mathrm{t}(528)=-2.84, \mathrm{p}<0.05)$.

The results show that there was a significant relationship between attitudes towards infidelity and education. Hence, the posthoc test of the Scheffe was used. The results indicate that there was a significant difference between the attitudes towards infidelity at the educational level of individuals (associate degree, bachelor's degree, master's degree and above).

\section{Discussion}

Attitude toward infidelity has emerged as a significant variable in lots of studies. People with easy approach toward infidelity are more likely to get involved in it $[4,6,7]$. In addition, Buunk and Bakker [8] found that attitude toward extra marital sex is an important index to predict the willingness toward it, because the beliefs and values encourage or prohibit the engagement in the infidelity relations. The aim of the present study was examining the psychometric properties and factor structure of the Attitude toward Infidelity questionnaire among married men and women in Tehran. When an instrument is returned from a culture or language to another language or culture, it is necessary to examine its psychometric properties. The findings of the current study suggest that this scale is properly valid and reliable to measure attitudes toward infidelity in Iran. The internal consistency of attitudes toward infidelity scale by using cronbach's scale was estimated 0.71 . These findings suggest that this instrument has a good internal consistency. This finding is consistent with Whatley findings [18]. He found the reliability of this instrument as 0.80 using cronbach's alpha. The obtained test-retest reliability $(0.87)$ was also desirable and satisfied.

In the present study, the first time confirmatory factor structure was used to investigate the attitude toward infidelity. The first time factor structure of attitudes toward infidelity had better fitness with observed data. Wittey study [39] also indicated an appropriate factor structure for the attitudes toward infidelity. The confirmatory factor structure, reliability and validity of attitudes toward infidelity scale were considered as acceptable to be used in research and clinical diagnosis. However, according to the results of attitude toward infidelity scale, this scale was reliable and valid to assess Iranian families and achieve steady and stable results.

In order to evaluate the divergent validity we used the lack of correlation between the total score of attitudes toward infidelity scale, the sub-scales of allport religious orientation, Kansas marital satisfaction and Enrich sexual satisfaction. As we know, the attitude toward infidelity scale examines the behaviors theoretically which is different with two subscales of internal and external religious orientation. So the negative correlation between this test and the previous two subscales in the present study would be regarded as an evidence for suitable divergent validity of this scale. In addition, negative correlation between the attitudes toward infidelity scale and Kansas marital satisfaction and subscale of Enrich sexual satisfaction showed that those who had easy approach to infidelity, experienced lower marital and sexual satisfaction and it implies that the scale of attitudes toward infidelity has divergent validity properly. In this regard Atkins et al [12] Previti and Amato's [39] studies showed that those who have lower marital satisfaction, more likely try to betray his/ her partner. Since sexual satisfaction is one of the main factors of the preservation and maintenance of marital relationship, if each of these factors in a relationship be impaired, the person feels fewer restrictions on his relationship and turned in to infidelity with more convenience.

The results of the interaction analysis of demographic variables with the scale showed that there is no significant correlation between age and attitudes toward infidelity. This means that the attitude towards infidelity has 
no difference at different ages. This finding is not consistent with the studies of Laumann et al. [13], Wiederman [5].

This finding may be due to the fact that the age factor cannot create a different attitude in the field of infidelity by itself, but it can be considered as a mediator factor only. On the other hand, the findings of this study suggest that there was a significant difference between gender and attitudes towards infidelity. Men had more easygoing attitudes towards infidelity compared to women. These findings are consistent with the results of Atkins et al [12], Laumann et al [13], Munsch [10], Petersen, Janet and Hyde [3] and Wiederman [5]. Besides, the current study showed a significant difference between educational level and attitudes toward infidelity. People with higher education had more easygoing attitudes toward infidelity. This finding is also consistent with the studies of Forste, Koray, and Tanfer [17] and Atkins et al [12].

It should be noted that some restrictions, limited the results of the study. First, using self-report instruments might encourage participants to use the methods of obtaining social approval and avoiding of the incompetence (rather than actual behavior) in this study. Second, the issues related to culture, ethnic and cultural influences related attitudes toward extramarital relations was ignored in this study. Third, the sample was consisted of married men and women in Tehran, people with other professions and social classes were not included in thestudy sample.

\section{Conclusion}

Finally, with regard to the goals of the Automatic Terminal Information Service (ATIS) scale for attitudes toward infidelity, it can be said that this scale is applicable for individuals or groups, in several cases, as a diagnostic instrument in the field of family counseling and couples therapy. This tool can be used for research purposes in regard to marriage, family and close relationships among different people.

\section{Acknowledgments}

It is appreciated from all persons that participated in this study. This research article has not received any financial support from any organization.

\section{Authors' contributions \\ Study design: HA \\ Data collection and analysis: MH, HA, MP \\ Manuscript preparation: HA, MP, SS \\ All authors have read and approved the final version}

\section{Conflict of Interest}

"The author declares that they have no competing interests."

\section{Availability of data and materials}

The datasets used and/or analyzed during this study are available from the corresponding author on reasonable request.

\section{References}

1- Johnson SM. Broken bonds: An emotionally focused approach to infidelity. J Couple Relatsh Ther2005; 4(2-3):17-29.

2- Brand RJ, Markey CM, Mills A, Hodges SD. Sex differences in self-reported infidelity and its correlates. Sex Roles2007; 57(1-2):101-9.

3- Petersen JL, Hyde JS. A meta-analytic review of research on gender differences in sexuality, 19932007. Psychol Bull2010; 136(1): 21-38.

4- Treas J, Giesen D. Sexual infidelity among married and cohabiting Americans. J Marriage Fam2000; 62(1):48-60.

5- Wiederman MW. Extramarital sex: Prevalence and correlates in a national survey. J Sex Res1997; 34(2):167-74.

6- Glass SP, Wright TL. Justifications for extramarital relationships: The association between attitudes, behaviors, and gender. J Sex Res1992; 29(3):361-87.

7- Thompson AP. Emotional and sexual components of extramarital relations. J Marriage Fam 1984; 46(1):35-42. 8- Buunk BP, Bakker AB. Extradyadic sex: The role of descriptive and injunctive norms. J Sex Res1995; 32(4):313-8.

9- Byers ES. How well does the traditional sexual script explain sexual coercion? Review of a program of research. J Psychol Human Sex1996; 8(1-2):7-25.

10- Munsch C. The effect of relative income disparity on infidelity for men and women. American sociological association. Press release retrieved October. 2010; 22: 2010.

11- Zilbergeld B. The new male sexuality: revised 
edition. New York: Bantam Books; 1999.

12- Atkins DC, Baucom DH, Jacobson NS. Understanding infidelity: correlates in a national random sample. J Fam Psychol2001; 15(4): 735-49.

13- Laumann EO. The social organization of sexuality: Sexual practices in the United States. USA, Chicago: University of Chicago press; 1994.

14- Lieberman B. Extrapremarital intercourse: Attitudes toward a neglected sexual behavior. J Sex Res1988; 24(1): 291-8.

15- Hansen GL. Extradyadic relations during courtship. J Sex Res 1987; 23(3): 382-90.

16- Smith TW. Attitudes toward sexual permissiveness: Trends, correlates, and behavioral connections. Sexuality across the life course. Edited by Alice S. Rossi. Chicago: University of Chicago press; 1994. pp: 63-97.

17- Forste R, Tanfer K. Sexual exclusivity among dating, cohabiting, and married women. J Marriage Fam1996; 58(1): 33-47.

18- Whatley M. Attitudes toward infidelity scale: Ed: D. Knox, ve C. Schacht) Choices in relationships. Belmont, California: Thompson Wadsworth publishing; 2008.

19- Howell D. Statistical methods for psychology.USA, Vermont: Wadsworth Cengage Learning; 2012.

20- Allport GW, Ross JM. Personal religious orientation and prejudice. J Pers Soc Psychol1967; 5(4): 432-43.

21- Mokhtari A, Allahyari A, Rasoolzade Tabatabei SK. Relationship between religious orientation and tension. Journal of Psychology2001; 5(17): 56-67.

22- Grover KJ, Russell CS, Schumm WR, Paff-Bergen LA. Mate selection processes and marital satisfaction. Fam Relat 1985; 34(3): 383-6.

23- Morris ML, Blanton PW. The influence of workrelated stressors on clergy husbands and their wives. Fam Relat1994; 43(2): 189-95.

24- Hunsley J, Pinsent C, Lefebvre M, James-Tanner S, Vito D. Construct validity of the short forms of the Dyadic Adjustment Scale. Fam Relat1995; 44(3): 231-7.

25- Schumm WR, Paff-Bergen LA, Hatch RC, et al. Concurrent and discriminant validity of the Kansas Marital Satisfaction Scale. J Marriage Fam1986; 48(2): 381-7.

26- Spanier GB. Measuring dyadic adjustment: New scales for assessing the quality of marriage and similar dyads. J Marriage Fam1976; 38(1): 15-28.

27- Norton R. Measuring marital quality: A critical look at the dependent variable. J Marriage Fam 1983; 45(1): 141-51.

28- Weigel DJ, Ballard-Reisch DS. How couples maintain marriages: A closer look at self and spouse influences upon the use of maintenance behaviors in marriages. Fam Relat1999; 48(3): 263-9.

29- Schumm WR, Nichols CW, Schectman K, Grigsby

CC. Characteristics of responses to the kansas marital satisfaction scale by a sample of 84 married mothers. Psychol Rep1983; 53(2): 567-72.

30- Olson D. Prepare/enrich counselor's manual. Minneapolis, MN: Life innovations. Inc; 1999.

31- Butzer B, Campbell L. Adult attachment, sexual satisfaction, and relationship satisfaction: A study of married couples. Pers Relatsh2008; 15(1): 141-54.

32- Fowers BJ, Olson DH. ENRICH marital Inventory: A discriminant validity and cross-validation assessment. J Marital Fam Ther1989; 15(1): 65-79.

33- Hayduk LA. Structural equation modeling with LISREL: essentials and advances. Boltimor and London: The johns hopkins press; 1988.

34- Jöreskog KG, Sörbom D. LISREL 8: User's reference guide. USA, Lincolnwood: Scientific Software International; 1996.

35- Bentler PM. Comparative fit indexes in structural models. Psychol Bull1990; 107(2): 238-46.

36- Tabachnick BG, Fidell LS. Using multivariate statistics. New York: Harper and row; 2006.

37- Hu Lt, Bentler PM. Cutoff criteria for fit indexes in covariance structure analysis: Conventional criteria versus new alternatives. Struct Equ Modeling1999; 6(1): $1-55$.

38- Campbell DT, Fiske DW. Convergent and discriminant validation by the multitrait-multimethod matrix. Psychol Bull1959; 56(2):81-105.

39- Whitty MT. Pushing the wrong buttons: men's and women's attitudes toward online and offline infidelity. Cyber Psychology \& Behavior2003; 6(6): 569-79.

40- Previti D, Amato PR. Is infidelity a cause or a consequence of poor marital quality? J Soc Pers Relat2004; 21(2): 217-30.

\footnotetext{
Copyright(C) 2016 ASP Ins. This open-access article is published under the terms of the Creative Commons Attribution-NonCommercial 4.0 International License which permits Share (copy and redistribute the material in any medium or format) and Adapt (remix, transform, and build upon the material) under the Attribution-NonCommercial terms.
} 